\title{
La biopolitique dans le plus simple appareil
}

\section{Grégory Salle}

\section{(2) OpenEdition}

Journals

\section{Édition électronique}

URL : http://journals.openedition.org/conflits/17968

DOI : $10.4000 /$ conflits. 17968

ISSN : $1777-5345$

Éditeur :

CCLS - Centre d'études sur les conflits lilberté et sécurité, L'Harmattan

\section{Édition imprimée}

Date de publication : 15 décembre 2010

Pagination : 107-117

ISBN : 978-2-296-54483-3

ISSN : 1157-996X

\section{Référence électronique}

Grégory Salle, «La biopolitique dans le plus simple appareil », Cultures \& Conflits [En ligne], 78 | été 2010, mis en ligne le 06 mars 2012, consulté le 30 mars 2021. URL : http://journals.openedition.org/ conflits/17968; DOI : https://doi.org/10.4000/conflits.17968 


\section{La biopolitique dans le plus simple appareil}

\section{Grégory SALLE}

Grégory Salle est chargé de recherche au CNRS, rattaché au Centre lillois d'études et de recherches sociologiques et économiques (CLERSÉ - CNRS/Université Lille 1). Membre du comité de rédaction de Cultures \& Conflits, il termine actuellement un ouvrage consacré à la question carcérale en France (2000-2009).

Olivier Razac, Histoire politique du barbelé, Paris, Flammarion, coll. «Champs essais », 2009 [2000] (2 2 ème éd. revue et augmentée), 240 p., préf. d'Alain Brossat.

"Seule une nature profondément sensible peut être assez réceptive à l'horreur pour parvenir presque à la définir en se contentant de la décrire. » (Allen Ginsberg)

\footnotetext{
Onfrontons les deux objets. Le premier est extérieurement d'un gris quasi $\checkmark$ uniforme ; dépourvue d'image, sa couverture est parcourue de traits verts et de caractères blancs. Ses 112 pages mesurent $160 \mathrm{~cm}$ sur 110, pour un poids de 90 g. Elles ont été publiées en 2000, alors que leur auteur était en pleine thèse. Le second objet, le successeur, est jaune vif et une barbe - de barbelé s'entend - orne sa couverture. Au dos, le texte de présentation a entièrement changé. Le nouvel éditeur (Flammarion ayant pris le relais des recommandables éditions La Fabrique ${ }^{1}$ ) annonce une version "revue et très largement augmentée » : la promesse, on le verra, est tenue. On y croise au passage le nom de Foucault, et l'ouvrage est en effet traversé par un dialogue avec l'auteur de Surveiller et punir. Le signataire de cette Histoire politique du barbelé est toujours philosophe, mais depuis son premier coup d'essai et coup de mâ̂tre, une petite décennie plus tôt, sa bibliographie s'est nettement étoffée, à commencer par l'ouvrage tiré de la thèse déjà évoquée ${ }^{2}$. Si le livre de 2009 est à peine plus haut que son prédécesseur, il est en revanche sensiblement plus

1. Razac O., Histoire politique du barbelé. La prairie, la tranchée, le camp, Paris, La Fabrique, 2000.

2. Razac O., La grande santé, Paris, Climats, 2006.
} 
épais et presque deux fois plus lourd, et l'on s'aperçoit en le feuilletant qu'il est rempli d'une typographie plus petite et plus dense. L'ouvrage remis sur le métier compte aussi une bonne dizaine d'illustrations. C'est un peu plus que dans la précédente version, bien que certaines d'entre elles aient été abandonnées en chemin. Parmi celles-ci, l'une montrait des couvertures de livres traversées du motif du barbelé, pour illustrer la façon dont celui-ci fonctionne comme emblème exprimant à lui seul l'oppression. Ironie du sort, cette nouvelle couverture le confirme tacitement. Le sous-titre originel (« La prairie, la tranchée, le camp »), lui, a disparu. Il est en fait devenu le titre de la première partie d'un livre qui en compte désormais trois au lieu de deux. La deuxième («Le barbelé et la gestion politique de l'espace ») a été augmentée, tandis qu'une nouvelle partie, intitulée «Les nouvelles délimitations de l'espace », prolonge et approfondit les réflexions de l'auteur sur l'investissement politique de l'espace en général et sa virtualisation en particulier.

Cette entrée en matière apparemment terre à terre est un simple clin d'œil à la matérialité de l'objet ici étudié autant qu'au matérialisme philosophique, ancré dans les sciences humaines et sociales, de l'auteur. Ce dernier sait restituer au lecteur juste ce qu'il faut du détail technique ou opérationnel des dispositifs qu'il analyse en les décrivant, dans le même mouvement. À cet égard, bien que les travaux de Bruno Latour ne soient pas ici mis à contribution, on songe parfois au fait qu'Olivier Razac serait tout désigné pour tenter de réaliser non une improbable et inutile synthèse, mais un programme FoucaultLatour, à la manière dont Mary Douglas parlait d'un programme DurkheimFleck. Au sens aigu des rapports de pouvoir de Foucault, il ajoute le goût particulier pour la description des objets, procédés et dispositifs contemporains de Latour. Certes, O. Razac récuserait certainement tout « amour des techniques » ${ }^{3}$, même mis au service d'une réflexion qui sait faire preuve d'un certain ludisme (il y a un sens du jeu ou une sorte de « gai savoir » qui, peut-être, rapproche les deux auteurs malgré la distance qui les sépare à bien d'autres égards); il faut dire que son objet d'étude n'invite pas à un tel sentiment. De plus, l'intention critique, dans tous les sens du terme, est ici évidente, même si elle ne s'affirme au grand jour que dans l'ultime phrase. Mais l'on ne peut s'empêcher de voir à l'œuvre, au moins en puissance, une certaine conciliation des styles de ces deux auteurs dans la manière avec laquelle $\mathrm{O}$. Razac décèle, dans une matière apparemment triviale, bien plus que ce qu'elle donne à voir immédiatement.

Le premier intérêt du livre d'Olivier Razac est là : dans l'idée astucieuse, pour ne pas dire géniale, de faire l'histoire d'un objet visiblement des plus banals, si ce n'est insignifiant, dénué en tout cas de toute noblesse. Quoi d'autre, au fond, qu'un misérable bout de ferraille hérissé de dards, dont la paternité revient officiellement à un obscur fermier de l'Illinois, suffisamment

3. Allusion à Latour B., Aramis ou l'amour des techniques, Paris, La Découverte, 1992. 
conscient de ses intérêts pour faire breveter (déjà !) son invention en 1874. Une chose sans dignité donc, si primitive qu'elle semble à peine mériter le nom de dispositif, même au sens plat du mot. Le livre montre qu'il en va, au contraire, tout autrement, et ne peut que saluer le caractère brillant, dans sa simplicité, de l'invention. Il en fait ensuite un objet d'étude aussi épineux intellectuellement qu'il l'est matériellement. Plus encore, il parcourt à travers lui, ou avec lui, une histoire aussi impressionnante que frappée du sceau du tragique. Car le barbelé ne se présente pas seulement comme rudimentaire, mais aussi comme répulsif, et ce, au-delà de sa vocation première. Il est partie prenante de trois des pires abjections historiques : ethnocide des Indiens, carnage de la Première Guerre mondiale, atrocité des camps. Au cours de la mal nommée « conquête de l'Ouest », il « crée les conditions de la disparition physique et culturelle de l'Indien » (p. 39). Lors de la (tout aussi mal nommée) « der des ders ", ses multiples qualités, de sa portabilité à sa quasi invisibilité, convertissent ce redoutable instrument en composante de la guerre de tranchées. Il devient ensuite constitutif des camps de la mort (« le camp, c'est les barbelés ») dans leur monstrueuse matérialité. Là, les barbelés ne garnissent pas seulement les enceintes, mais ils délimitent au sein même des camps des seuils différenciés et des subdivisions hiérarchisées. Au-delà du matériau de construction, le barbelé est ainsi « l'élément central d'une gestion totalitaire de l'espace» (p. 68). Somme toute, avec le plus petit, le barbelé, O. Razac fait voir le plus grand. C'est ainsi qu'après avoir revisité « trois des plus grandes catastrophes de la modernité », il esquisse une " géographie politique du barbelé » contemporaine dont les critères de démarcation (violence objective, rapport subjectif à la violence, force négative du symbole) dessinent une cartographie mondiale insolite.

Ce sens de l'objet - dont il scrute tant les effets matériels que la charge symbolique - a grandement contribué au succès de la première version du livre, traduite en plusieurs langues. Le lecteur dépourvu de ce genre d'intuitions - on peut classer dans un genre voisin l'histoire de la voiture piégée de Mike Davis ${ }^{4}$ - comme du talent nécessaire à leur développement peut convertir sa jalousie en un sentiment plus fécond, s'interrogeant sur l'origine de l'idée. Déclic ou fruit d'une lente maturation ? Est-ce une lecture, un film, une conversation qui a mis l'auteur sur la piste ? La lecture de The Wire that Fenced the West, livre plusieurs fois mis à contribution de Henry et Frances Mc Callum, publié en 1965 aux obscures (mais ici toutes désignées !) presses universitaires de l'Oklahoma, a-t-elle été à l'origine de l'enquête, ou en a-t-elle été plutôt un jalon important une fois celle-ci entamée ? Quoiqu'il en soit, en s'attardant sur cet artefact, non seulement $O$. Razac faisait alors preuve d'un flair intellectuel qui fait largement défaut à la science politique mainstream mais, mieux encore, il prenait à revers sa propre école de pensée ou, disons, des courants théoriques dont on peut présumer qu'ils sont pour lui des sources

4. Davis M., Petite histoire de la voiture piégée, Paris, Zones/La Découverte, 2007. 
d'inspiration privilégiées. L'auteur ne part pas dans ce livre sur la piste des «sociétés de contrôle » dessinées par Deleuze, bien qu'il la croise d'une certaine manière en fin de parcours. Marque de lassitude ou contre-pied volontaire, le célèbre " post-scriptum » deleuzien n’est même pas mentionné. Il ne s'intéresse pas ici essentiellement (il l'a fait par ailleurs, comme en témoignent ses travaux sur le GPS, le bracelet électronique ou les armes dites « de neutralisation momentanée »5) à la sophistication technologique des outils de la domination, même si les derniers développements lui sont consacrés. Non : il se focalise sur le plus simple appareil. Et il le déplie, il l'explique au point d'en tirer des thèses générales, concernant non seulement le rôle historique de cet outil, mais plus largement la gestion politique de l'espace, et corrélativement les formes d'encadrement du vivant. C'est là qu'entre en scène la biopolitique ; on y reviendra.

Ce premier tour de force se double d'un second, sur un autre mode paradoxal. C'est déjà beaucoup d'inscrire l'étude du barbelé dans la perspective plus large d'une « histoire des technologies de délimitation de l'espace » (p. 85) sous l'angle de leur virtualisation (par quoi il faut entendre non une réalité amoindrie mais une rationalisation du rapport entre efficacité et coût), laquelle est définie par cinq caractéristiques (effacement/allègement, mobilité, souplesse, discrétion, réactivité). Reste que dans ce cadre, on aurait pu penser que le barbelé marquerait le stade ultime du processus, et donc le point d'aboutissement de l'analyse. Quoi de plus ténu, en effet, qu'un fil de fer barbelé ? Or, l'auteur projette au-delà : le barbelé est ici une borne intermédiaire, certes cruciale, mais non finale, " une étape technologique décisive dans une histoire de la virtualisation des délimitations » (p. 143). L'euphémisation matérielle de l'aménagement spatial des rapports de pouvoir ne s'arrête pas avec le barbelé. Il a vocation à être dépassé ou remplacé « par des techniques plus éthérées, par des dispositifs plus furtifs traçant des limites immatérielles » (p. 25) ${ }^{6}$. Après avoir passé en revue, de Gaza à Guantánamo en passant par « l’Europe barbelée ", les multiples usages contemporains (on le trouve y compris faire office de cordon sanitaire à l'usage des nantis, déployé autour de leurs quartiers résidentiels) de cet outil ô combien tenace, O. Razac signale en effet la limite que constitue son coût symbolique. Associé aux horreurs du siècle, le barbelé est devenu l'expression ramassée de l'oppression la plus absolue (un idéogramme, propose Alain Brossat dans une remarquable préface), véhiculant tout un imaginaire de violence et de terreur. Il est dès lors mal ajusté à la sensibilité contemporaine, du moins sous les contrées les mieux loties. Alors surgissent des frontières aux abords plus avenants, jusqu’à une « haie défensive tressée

5. Cf. Razac O., Avec Foucault, après Foucault. Disséquer la société de contrôle, Paris, L'Harmattan, 2008 ; L'utilisation des armes de neutralisation momentanée en prison, CIRAP, $\mathrm{n}^{\circ} 5,2008$.

6. L'auteur s'est par ailleurs penché sur des types de techniques quasi imperceptibles : cf. par exemple Razac O., "La musique des délimitations de l'espace », in Avec Foncault, après Foucault, op. cit., p. 63-73. 
naturelle ", clôture végétale réalisant la prouesse de marier les nouvelles normes écologiques et esthétiques...

C'est par le sens de l'objet comme par le goût de la problématisation que la première version du livre comptait parmi les travaux - relativement rares, en dépit des apparences - méritant sérieusement le qualificatif de foucaldien, loin des pâles copies et des resucées paresseuses ou contrefaites. Si l'on peut rapprocher Histoire politique du barbelé de Surveiller et punir sur plusieurs points (par exemple l'iconographie déjà évoquée), une sorte de symétrie est en même temps repérable dans la stratégie d'exposition. Dans le livre de 1975, l'objet carcéral stricto sensu annoncé par le sous-titre (Naissance de la prison) était essentiellement traité dans le dernier chapitre («Le carcéral »); le cas de la prison n'était en quelque sorte que la pointe émergée de l'iceberg, l'avènement de l'âge des disciplines, lequel nécessitait d'être exposé préalablement. Le mouvement est ici inverse : c'est du barbelé que l'on part, et c'est progressivement que son étude s'inscrit dans l'ambition plus vaste d'une histoire politique et technique des délimitations de l'espace, jusqu'aux procédés de sélection et d'exclusion soft, voire indétectables. Dans les derniers développements, le barbelé en tant que tel tend du reste à s'effacer, comme si l'écriture rendait compte en elle-même de la dynamique historique de l'objet, voué à la caducité, du moins dans certains lieux et en certaines circonstances. Comme ce fut le cas pour Foucault, les historiens sourcilleux pourront blâmer la désinvolture vis-à-vis des règles conventionnelles (si tant est qu'elles existent...) de l'écriture historique, ou simplement regretter la dilatation, ou la dilution, de l'objet, dans la mesure où la dernière partie de l'ouvrage aborde une vaste gamme de techniques de filtrage ou de contrôle (des caméras de surveillance aux caisses électroniques des supermarchés), parfois incarnées (vigiles et autres médiateurs). De ce point de vue, cette partie du livre est moins serrée et, sans doute, plus fragile que les précédentes. Mais c'est aussi qu'elle n'est pas fermée sur elle-même, ayant vraisemblablement une fonction exploratoire ou programmatique pour d'autres travaux. En outre, accoler le barbelé à des choses qui n'ont a priori rien à voir est aussi un moyen heuristique pour bousculer les associations spontanées (c'est-à-dire héritées) du sens commun. Qu'on pense par exemple aux commentaires de Siegfried Kracauer à propos de l'auteur du Procès: «Les récits de Kafka décrivent un univers d'horreur qui rappelle de près les organisations humaines dont les triomphes sont les tranchées, les barbelés et les projets financiers les plus élaborés» " ${ }^{7}$.

La discussion avec Foucault s'effectue tout au long du livre mais par touches successives, jusqu'à un épilogue sur la géolocalisation qui prolonge en quelques pages stimulantes les modèles de quadrillage développés notamment dans Sécurité, territoire, population, le cours au Collège de France de 1977-

7. Cité in Traverso E., Siegfried Kracauer. Itinéraire d'un intellectuel nomade, Paris, La Découverte, 2006, p. 45. 
1978. O. Razac avance l'idée que le porteur d'un bracelet électronique, «certainement la virtualisation la plus aboutie des délimitations de l'espace » (p. 231), tient à la fois du lépreux (exclu et refoulé), du pestiféré (inclus et cantonné) et du convalescent, dont la mobilité est soigneusement contrôlée, selon le modèle de la variole ${ }^{8}$. Qu'elle reprenne directement ou non telle ou telle notion foucaldienne, la discussion est ingénieuse et rigoureuse, qu'il s'agisse des développements consacrés à la notion d'hétérotopie qui ouvrent la deuxième partie, du rapprochement inattendu mais suggestif sur les effets panoptiques d'un dispositif barbelé-surveillance, ou de la caractérisation d'un « diagramme stratégique » au principe de différentes modalités de délimitation de l'espace ${ }^{9}$.

Il en va de même pour les développements relatifs à la biopolitique (et de son envers ou de son pendant thanatopolitique). O. Razac s'explique d'ailleurs (p. 145-146) sur son rapport à la notion, sur les précautions d'usage qu'elle requiert, dans la mesure où elle fait l'objet d'usages relativement changeants de la part de Foucault en fonction des problèmes en jeu. Pour autant, il se tient à mille lieux de l'exégèse bavarde comme de l'incantation conceptuelle. En mettant le concept à l'épreuve d'un cas d'étude spécifique autant que d'un problème déterminé, il contribue à en réactiver la saveur tout en lui restituant sa fonction d'outil d'analyse. Il tâche ainsi de différencier et de caractériser, de façon certes discutable, une biopolitique «totalitaire » (comme pour la biopolitique, l'inflation discursive pas toujours contrôlée autour du terme aurait peut-être mérité quelques précisions préalables sur son emploi) et une biopolitique « libérale ». L'essentiel est qu'il ne s'agit pas par là de garnir des considérations générales, mais de faire jouer des concepts au service d'une thèse : « le barbelé est l'outil paradigmatique d'une gestion politique de l'espace qui se caractérise à la fois par la radicalisation, l'animalisation et la hiérarchisation [...] le barbelé est bien un outil biopolitique de gestion de l'espace, plus précisément, il est l'outil le plus caractéristique de la biopolitique dans ses manifestations spatiales» (p. 86 ; p. 108-109). Tracer la limite entre vivre et mourir, rejeter le mauvais côté dans l'infra-humain ou la sous-existence (le champ versus le désert, le troupeau versus les bêtes sauvages), mais aussi sortir du schéma binaire pour classer la vie sur une échelle graduée : on est au cœur du sujet. Les thèses avancées ou ébauchées le sont avec précision et un sens aigu de la dialectique, comme lorsque le barbelé est conçu comme «le pli qui sépare et relie

8. Sans calquer le célèbre « grondement de la bataille » qui ponctuait, sans l'achever, Surveiller et punir, l'auteur suggère à sa manière de braquer le regard sur les luttes bruyantes ou sourdes qui composent notre actualité. À ce niveau, les dépassements successifs retracés dans le livre se trouvent eux-mêmes dépassés dans la mesure où, même virtualisées, les délimitations deviennent, suggère $\mathrm{O}$. Razac, un enjeu subalterne des rapports de pouvoir présents.

9. On peut avancer au passage que, si le barbelé a quelque chose de « disciplinaire », la notion peut renvoyer non seulement à Foucault mais aussi peut-être à Max Weber, en un sens bien distinct du précédent, teinté notamment de probabilisme. La discipline définit alors la " chance » d'obtenir " une obéissance prompte, automatique et schématique » (Weber M., Economie et Société, t. 1, Paris, Agora Pocket, 1995 [1956], p. 95.). Il semble bien que le barbelé provoque spontanément une réaction (répulsive) de ce type. 
les espaces thanatopolitiques et biopolitiques » (p. 121). De manière générale, O. Razac rapporte de façon convaincante la « virtualisation des délimitations » à une «inversion des principes de l'investissement politique de l'espace » (un surcroît de pouvoir avec une économie de moyens, la discrétion et non le spectacle), à une norme régulatrice plutôt qu'à la loi prohibitrice ou déontique, et par extension au type de gouvernementalité dit biopolitique où, en l'occurrence, il est moins question de «fixer des frontières » que de «suivre des mouvements ", au sens actif ou interventionniste de réguler des circulations, de canaliser des flux.

« La confirmation et le rejet ne sont que deux manières d'abuser d'un penseur ", avertit Peter Sloterdijk, suggérant plus tôt que l'importance de Foucault dans l'histoire de la pensée n'était pas encore comprise à sa juste valeur 10. À l'occasion, donc, O. Razac discute les thèses foucaldiennes, dans le sillage du concept de démocratie immunitaire élaboré par Alain Brossat pour mettre en évidence un mouvement historique parallèle à celui des disciplines : le souci croissant, certes conditionnel, d'une protection de principe des corps, personnes et opinions contre la violence ou la douleur ${ }^{11}$. C'est à l'aune de ce paradigme immunitaire que le barbelé est de plus en plus insupportable, y compris seulement visuellement, du moins dans certains lieux. Une acception réactualisée de la notion de pastorat sert ici d'échangeur conceptuel. O. Razac insiste bien sur la double nature, indissociable, de l'interface que constitue le barbelé. De même qu'il souligne que son efficacité n'est pas seulement spatiale, mais aussi temporelle (retarder la progression de l'ennemi, par exemple), il rappelle que la face dissuasive, la plus évidente, celle qui à la limite trace le partage entre la vie et la mort, coexiste avec la face accueillante, protectrice, celle qui enclot la prospérité. Revisitant l'origine de l'outil, destiné à enceindre le bétail, il fait du barbelé «l'outil pastoral par excellence» (p. 107). Pastoral et donc biopolitique ici, car « un troupeau, ça se respecte» (p. 115) ${ }^{12}$. Par ailleurs, en s'appuyant sur des pistes tracées par Foucault mais pour s'en démarquer, l'auteur traite frontalement de la question délicate des camps pour en faire des lieux d'entrelacement extrême du pouvoir souverain et de la gestion biopolitique : «Le camp est le lieu de la superposition de la double polarité pastorale. Ce sont des usines et des lieux de désolation. [...] Si le barbelé devait être un symbole, ce ne serait pas celui de la violence politique dans sa dimension barbare et archaïque mais plutôt celui de cet entrelacement proprement

10. Sloterdijk P., Ni le soleil ni la mort, Paris, Hachette, 2003 [2001], p. 119 et p. 16.

11. Il ne s'agit là que d'un résumé très réducteur : cf. Brossat A., La démocratie immunitaire, Paris, La Dispute, 2003.

12. On pourrait toutefois objecter qu'on perd ici au passage en précision conceptuelle, dans la mesure où chez Foucault, pastorat et biopolitique sont volontiers conçus comme deux régimes distincts, sinon opposés, de gouvernementalité, dans la mesure où le premier s'applique à la conduite des âmes, plutôt qu'à la gestion des corps, de la vie ou de la population au sens moderne. Ces deux notions émergent en fonction de problèmes distincts, et répondent aussi à une périodisation différente. 
moderne entre la désolation et la productivité, l'abandon et l'efficacité, le meurtre et la surprotection » (p. 120 et p. 141).

Si l'analyse des trois moments paroxystiques est passionnante et décapante, c'est plus généralement que les travaux sur la circulation des techniques trouveront là, à condition qu'ils s'intéressent aux rapports de pouvoir, un cas d'étude riche d'enseignement. Foucault faisait remarquer que la formule pénale de l'emprisonnement " a été finalement et actuellement reprise dans tous les contextes politiques et sociaux. Cela a été une si formidable invention, et si merveilleuse, qu'elle s'est répandue presque comme la machine à vapeur et est devenue une forme d'encadrement général de la plupart des sociétés modernes, qu'elles soient capitalistes ou qu'elles soient socialistes » ${ }^{13}$. Le succès foudroyant et universel du barbelé fournit sûrement une meilleure analogie encore avec l'invention de Papin et Watt. Un angle mort demeure : celui des périodes de transition. Comment et par qui le barbelé a-t-il été importé en Europe et en Russie soviétique ? Comment s'est-il retrouvé, alors qu'il n'était encore qu'un fil lisse, mais déjà organisé en réseau, à servir pendant la guerre franco-prussienne de 1870 ? Comment a-t-il migré en URSS pour encadrer les convois en partance pour la Sibérie ? Et d'ailleurs s'agit-il forcément d'importations, ou des inventions locales simultanées d'un outil similaire sont-elles envisageables ? Plus enfouis, moins saillants, ces moments de développement demeurent encore obscurs dans le détail.

On se demande au passage pourquoi l'auteur s'en tient à parler d'histoire et non de généalogie. Comme le suggère A. Brossat dans sa préface, la perspective de cette Histoire politique du barbelé paraît en effet satisfaire les critères posés par Foucault, même si elle est peut-être insuffisamment " grise ", malgré les pépites documentaires dénichées à la bibliothèque du musée des Invalides ${ }^{14}$. Avant tout, l'auteur part bien d'une préoccupation présente, tout en évitant de succomber aux biais d'une lecture linéaire et téléologique. Il n'est pas à la quête d'un point d'origine ou d'une fonction essentielle, mais s'attache à repérer les accidents, les discontinuités, la part de contingence. S'il indique d'emblée que « le fil de fer barbelé n'a jamais été un simple outil agricole [et] est immédiatement devenu un outil politique d'une grande importance » (p. 23), il ne suggère pas pour autant que dès son invention, il était voué à garnir voire à créer les camps. La démonstration est au contraire attentive aux devenirs de cet outil, ou en termes plus sociologiques, à ses usages. Elle fait bien la part des choses entre le déterminisme technique et les appropriations circonstanciées. Dans cette perspective, O. Razac souligne tant les propriétés quasi immuables de l'objet (économique, efficace, multifonctionnel, résistant, discret, modulable...), que ses déclinaisons (il existe différents types de fils,

13. Foucault M., Dits et Écrits, t. II, Paris, Gallimard, 1995, p. 796.

14. Foucault M., « Nietzsche, la généalogie, l’histoire », in Balaudé J-F., Wotling P. (dir.), Lectures de Nietzsche, Paris, Le livre de poche, 2000, p. 13-30. 
variables selon leur épaisseur, leur résistance, la distance qui sépare leurs ronces ; on passe du barbelé originel au barbelé dit rasoir, etc.), ainsi que la pluralité de ses reprises et des déplacements dont elles résultent. C'est tantôt l'outil lui-même qui est perfectionné (ainsi son aménagement réticulaire ou son électrification), tantôt ses lieux et domaines d'application qui s'étendent ou se transforment, tantôt les agencements dans lesquels il est pris qui mettent en valeur telle ou telle de ses caractéristiques. Par exemple, avec les camps, sa discrétion, l'absence de traces qu'il laisse, a pris une nouvelle dimension, vertigineuse : «À Treblinka, le barbelé a contribué à la disparition de centaines de milliers de personnes, il a aussi servi à tenter de faire disparaître cette disparition » (p. 152).

De Foucault - encore lui - on retrouve aussi dans ce livre l'absence d'académisme dans le choix des sources. Sans hiérarchiser a priori les références (sans distinction en particulier entre la littérature à prétention scientifique et la littérature dite de fiction), O. Razac fait flèche de tout bois, d'un ouvrage de Foch à un extrait de la revue L'Horticulture française en passant par des rapports de diverses natures ou des brochures d'administrations ou d'entreprises. Selon les besoins de la démonstration, il cite des opus militaires comme des classiques de la littérature mondiale, témoignages de rescapés des tranchées (Barbusse, Dorgelès, Jünger) ou des camps (Antelme, Levi). Il nourrit son travail d'études historiques et de réflexions philosophiques, mais fait aussi référence à des œuvres cinématographiques. Plus on se rapproche de la fin, c'està-dire plus on colle à l'époque contemporaine, plus les sources sont composées d'articles de presse et de sites Internet. Cet aplatissement des sources n'est pas en soi un problème, il est au contraire ajusté au problème qui guide le livre. En revanche, une historicisation et/ou une sociologisation des références (qui parle exactement ? dans quel contexte ? quelle est la nature exacte du support ? etc.) auraient parfois pu être enrichissantes. De plus, même sans gloutonnerie excessive en la matière, et en sachant bien qu'il est impossible de tout lire (encore moins de tout lire sérieusement), on peut regretter que la bibliographie ne soit pas plus étoffée, que ce soit d'un point de vue général (l'absence de confrontation avec les thèses de Henri Lefebvre ou, plus près de nous, de David Harvey sur la dimension spatiale des rapports de domination) ou sur tel ou tel point abordé ${ }^{15}$. Dans un autre genre, il aurait peut-être été instructif (ceci dit à tout hasard) d'aller voir du côté de la production culturelle «populaire », de la bande dessinée (Lucky Luke) aux séries télévisuelles (Deadwood).

15. On pense par exemple, lorsque l'on croise la question du filtrage aéroportuaire ou la figure du checkpoint, respectivement à Linhardt D., "L'économie du soupçon ", Genèses, n 44 , 2001, p. 76-98 et à Ritaine E., «La barrière et le checkpoint : mise en politique de l'asymétrie », Cultures $\mathcal{E}$ Conflits, $\mathrm{n}^{\circ} 73,2009$, p. 15-33. Certains auteurs sont aussi cités à partir de textes «mineurs » qui ne permettent peut-être pas de les mobiliser pleinement : c'est par exemple le cas de Marc Bernardot (cf. Camps d'étrangers, Bellecombe-en-Bauges, éd. du Croquant, 2008). 
Mais pointer d'éventuels et douteux « manques » est à la fois facile, vain et présomptueux, surtout si cela dispense de prendre la mesure du travail accompli et des pièges évités. Par exemple, l'auteur se défend sans équivoque ${ }^{16} \mathrm{de}$ sous-entendre que le processus de virtualisation qu'il décrit est unilatéral et hégémonique, ne serait-ce que parce que la persistance du barbelé, si robuste et si fragile, découle des agencements qu'il compose avec d'autres éléments. On pense ici à l'analyse de la prolifération des «murs» (lesquels sont volontiers garnis de barbelés) par Wendy Brown - qui aurait grandement bénéficié de la lecture d'O. Razac -, dans laquelle elle lit la défaite de la souveraineté, et non sa recrudescence ${ }^{17}$. On peut sur certains points établir des rapprochements intéressants : le succès paradoxal de procédés archaïques à leur manière propre (l'anachronisme du mur tenant, par contraste avec le barbelé, à son aspect massif, monolithique, inamovible), la grande variété de leurs usages ou de leurs cibles, l'importance de leur dimension « théâtrale », celle de la mise en scène de l'apparence du pouvoir, etc. Wendy Brown se réfère à plusieurs reprises à Mike Davis et, en effet, on trouve chez l'auteur de Ecology of Fear de quoi alimenter la réflexion sur la matérialisation spatiale de l'exercice du pouvoir, y compris le moins subtil. On trouvera difficilement partage plus clair entre la vie et la mort («touchez la clôture et vous êtes mort ») que la ceinture électrifiée de la prison fédérale de haute sécurité de Calipatria en Californie : "clôture électrique menaçante, haute de presque cinq mètres, prise entre deux grillages à mailles losangées ordinaires. Chacun des quinze brins individuels du câble est parcouru par 5000 volts fournis par le barrage Parker; environ dix fois la tension reconnue comme mortelle» 18 . Une clôture anti-évasion qui, pourtant, n'est pas exempte d'attributions biopolitiques inattendues, après l'indignation exprimée par des associations de défense des animaux devant le spectacle navrant d'oiseaux pris au piège. Si son effet potentiellement létal sur les humains n'a guère fait débat, en revanche elle est désormais « la seule clôture de la mort au monde à l'épreuve des oiseaux et écologiquement correcte $\gg 19$.

16. «Il ne faudrait pas penser pour autant que le processus de virtualisation signifie une disparation des séparations traditionnelles, de même que la biopolitique n'a pas simplement remplacé la souveraineté. Il n'y a d'ailleurs même pas d'opposition, mais plutôt la création d'un continuum, de la muraille la plus massive à la délimitation la plus éthérée » (p. 144).

17. Brown W., "Souveraineté poreuse, démocratie murée », La revue internationale des livres et des idées, $\mathrm{n}^{\circ}$ 12, 2009, p. 30-35.

18. Davis M., Au-delà de Blade Runner. Los Angeles et l'imagination du désastre, Paris, Allia, 2006. "Ouverte en 1993, Calipatria est une prison de "niveau 4", c'est-à-dire de sécurité maximum, qui accueille aujourd'hui $10 \%$ des meurtriers condamnés en Californie, soit 1200 hommes. Pourtant le poste de garde de l'entrée principale est vide, comme dix des douze miradors du périmètre. Si l'étonnante absence de surveillance traditionnelle donne une impression de négligence, c'est une politique concertée » (p. 120-121, je souligne). On retrouve ici le principe utilitariste qui guide les métamorphoses des dispositifs sécuritaires.

19. Ibid., p. 123. 
Ce trait d'humour noir nous amène enfin à la qualité du style, qui n'est pas le moindre atout du livre. Même lorsqu'elle aborde les sujets les plus terrifiants, la plume ne cède pas à l'émotivité. L'écriture tend au contraire à être froide ou détachée. Parfois, $\mathrm{O}$. Razac préfère donner la parole à un témoin qui, en une phrase, exprime l'indicible. Ce pli clinique de l'écriture est, sans doute, un gage de précision. C'est aussi une voie particulière pour toucher la sensibilité du lecteur, comme le suggère la citation d'Allen Ginsberg (parlant de William Burroughs) citée en épigraphe. Autant qu'il sait faire preuve de nuance, $\mathrm{O}$. Razac sait d'ailleurs aussi forcer le trait, et ponctuer un chapitre par une phrase qui claque comme un coup de fouet ${ }^{20}$. Il évite cependant les effets de manche rhétoriques et la surenchère conceptuelle. On pourrait se risquer à dire que, comme son objet, $\mathrm{O}$. Razac vise dans sa démonstration le maximum d'efficacité avec une certaine économie de moyens. En outre, son écriture passe adroitement du général au particulier et réciproquement, de telle utilisation du barbelé à des développements inspirés par Tocqueville ou portant sur le statut et les motifs de la guerre de 14-18. On a déjà évoqué la belle préface, à la fois panoramique et personnelle, d'Alain Brossat - comme pour toutes les préfaces, elle peut se lire avantageusement après la lecture du livre proprement dit, comme un écho final à la lecture plutôt que comme une mise en condition. Dans sa préface à Avec Foucault, après Foucault, ce dernier parlait, à propos d'Olivier Razac (qui fut, sauf erreur, son thésard) de la " grâce du chercheur " : c'est bien de cela qu'il s'agit.

20. "C'est précisément ce que les "citoyens" protégés des États biopolitiques ne peuvent jamais vraiment oublier tant qu'ils perçoivent leurs espaces privilégiés comme clôturés par des barbelés ou des outils équivalents. Ils ne peuvent oublier qu'ils sont des bêtes de troupeau toujours susceptibles d'être rejetées comme des bêtes sauvages. C'est pourquoi aussi ce qui leur tient lieu de préoccupations politiques ne concerne pas tant leur participation à la vie de la cité que la question de savoir de quel côté des barbelés ils se trouvent » (p. 123). 